\title{
GRAINE project: Flight data analysis of balloon-borne experiment in 2015 with emulsion gamma-ray telescope
}

\author{
Keita Ozaki ${ }^{1 *}$ for the GRAINE collaboration ${ }^{1,2,3,4,5,6}$ \\ ${ }^{1}$ Kobe University, ${ }^{2}$ ISAS/JAXA, ${ }^{3}$ Nagoya University, ${ }^{4}$ Okayama University of science, ${ }^{5}$ Aichi \\ University of education, ${ }^{6}$ Utsunomiya University \\ E-mail: ozaki@radix.h.kobe-u.ac.jp
}

\begin{abstract}
GRAINE is a balloon-borne emulsion $\gamma$-ray telescope experiment to observe cosmic $\gamma$-ray with precise angular resolution and polarization sensitive, covering the energy range from $10 \mathrm{MeV}$ to $100 \mathrm{GeV}$. We performed the balloon-borne experiment at Alice Springs in Australia in 2015 with second model of the emulsion $\gamma$-ray telescope. Our purpose of this experiment is to detect the bright $\gamma$-ray source (vela pulsar) and to demonstrate angular resolution of our telescope. The balloon flight and recorvery of the payload were successfully performed. Current status of the flight data analysis from the emulsion chamber and the attitude monitor is presented.
\end{abstract}

The 34th International Cosmic Ray Conference,

30 July- 6 August, 2015

The Hague, The Netherlands

\footnotetext{
* Speaker.
} 


\section{Introduction}

Cosmic $\gamma$-ray observation can explore the high energy phenomena in space.

The $\gamma$-ray astronomy is progressing by Fermi and AGILE, and Fermi has detected over 3000 sources based on the first four years of science data[1]. However, for examples, observations of supernova remnant, galactic center, or $\mathrm{GeV} \gamma$-ray flare from $\mathrm{Crab}$, are still many tasks due to poor angular resolution. Next generation $\gamma$-ray telescope should be achieved higher angular resolution and polarization sensitive.

We advance the GRAINE project, balloon-borne emulsion $\gamma$-ray telescope to observe the $10 \mathrm{MeV}-100 \mathrm{GeV}$ comic $\gamma$-rays. Nuclear emulsion is a three-dimensional charged particle tracking detector with sub-micron position accuracy. Nuclear emulsion can be suppressed multiple coulomb scattering by measuring the beginning of electron-pair, higher angular resolution for $\gamma$-ray can be achieved. Nulcear emulsion also can be detected the linearly polarization of $\gamma$-ray by measuring the azimuthal angle of electron-pair. Therefore, GRAINE can be achieved higher resolution imaging e.g. SNRs and galactic center, and also polarimetry for pulsars and blazers.

For the first step, balloon-borne experiment was performed at Taiki Aerospace Research Field (TARF) in Japan in 2011. Flight duration was 4.3hours (1.6hours at $35 \mathrm{~km}$ ). In this experiment, We have developed the small-type $\left(125 \mathrm{~cm}^{2}\right.$ aperture area) emulsion $\gamma$-ray telescope, daytime star camera as an attitude monitor, GPS, and pressure gauge, and they were loaded on the balloon gondola. We demonstrated that our equipments were working well, and we observed atmospheric $\gamma$-ray background[2].

For the second step, balloon-borne experiment was performed at Alice Springs in Austrarila in 12th May 2015. In the second balloon-borne experiment, we have developed middle-type $\left(3780 \mathrm{~cm}^{2}\right.$ aperture area) emulsion telescope[3], and our purpose of this experiment is to detect the bright $\gamma$-ray source (vela pulsar) and to demonstrate telescope's angular resolution. Current status of the flight data analysis from 2015 balloon-borne experiment is described.

\section{Balloon experiment in 2015}

\subsection{Instrument}

\subsubsection{Emulsion chamber}

Figure 1 shows the structure of the emulsion chamber loaded on the GRAINE-2015 balloonborne experiment. Aperture area of one emulsion chamber is $37.8 \mathrm{~cm} \times 25.0 \mathrm{~cm}$. The emulsion chamber consists of alignment part, converter part, time-stamper part, and calorimeter part. We developed four emulsion chambers, so total aperture area is $3780 \mathrm{~cm}^{2}$. The alignment part consists of 3 handmade emulsion films. One emulsion film is $320 \mu \mathrm{m}$ thickness. The converter part consists of 100 handmade emulsion films and its thickness is $320 \mu \mathrm{m}$ per film. Total thickness and radiation length is $32.0 \mathrm{~mm}$ and $0.54 \mathrm{X}_{0}$, respectively. The time-stamper part consists of 8 handmade emulsion films, which were put on the multi-stage shifter[4][5]. 6 emulsion films are $264 \mu \mathrm{m}$ thickness per film and 2 emulsion films are $124 \mu \mathrm{m}$ thickness per film. The calorimeter part consists of the sandwich structure of 16 handmade emulsion films with $270 \mu \mathrm{m}$ thickness per film and 15 stainless plates with $1 \mathrm{~mm}$ thickness per plate. Total thickness and radiation length is $19.3 \mathrm{~mm}$ and $0.90 \mathrm{X}_{0}$, respectively. All parts of emulsion films were kept the structure by vacuum packing. 


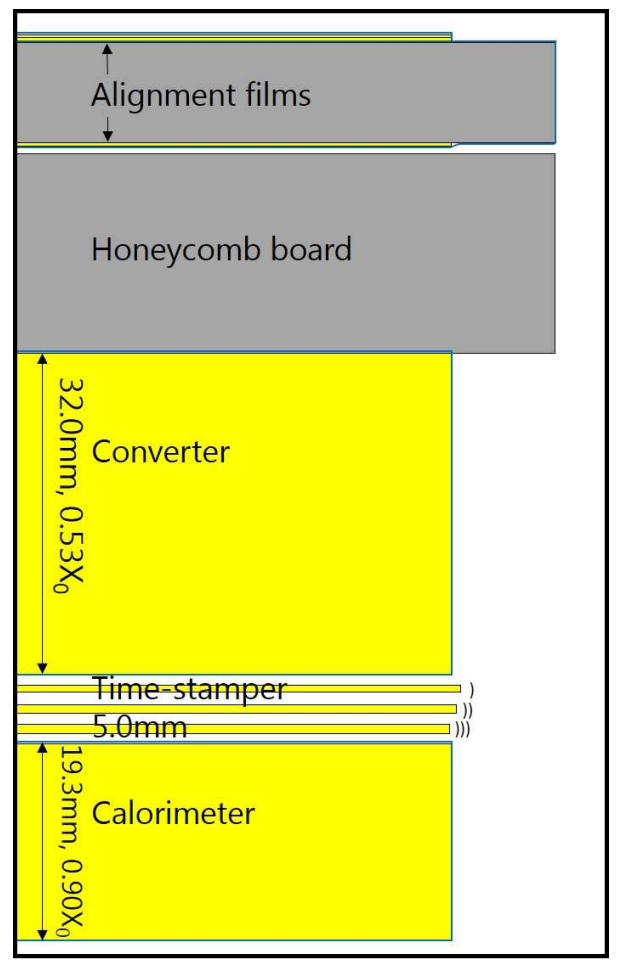

Figure 1: The structure of one emulsion chamber is shown (side view).

\subsubsection{Attitude Monitor}

We have developed the daytime star cameras as an attitude monitor. The $\gamma$-ray direction can be reconstructed to the celestial sphere by combining the timing and the attitude information. Three star cameras were loaded on GRAINE-2015 gondola as shown in Figure 2. Daytime star camera consists of CCD camera, lens, red-filter (transmits above $0.69 \mu \mathrm{m}$ ), and $66 \mathrm{~cm}$ length hood made of vinyl chloride. Raw images were transfered via Ethernet cable to CPU board (PCI-104), and saved to onboard SSD.

\subsubsection{Pressure vessel}

We have developed the fabric-type pressure vessel in order to keep vacuum packing of the emulsion chamber at balloon altitude[6]. The pressure vessel consists of inner layer which is natural rubber to keep air tightness and outer layer which is polyvinyl chloride fiber to keep its shape. Differential pressure of the vessel was managed about 0.3 atmospheric pressure by controlling a pressure regulating valve.

\subsection{Balloon flight}

The balloon was launched at Alice Springs in Australia in 12th May 2015 at 6:33 local time (UTC+0930). The flight path and the time variations of the balloon's altitude from GPS data are shown in Figure 3. Level flight at $37.2 \mathrm{~km}$ was started at 8:50. The operation of the time-stamper was finished at 20:02. After that, the gondola was cut off at 20:22. The gondola was landed at 


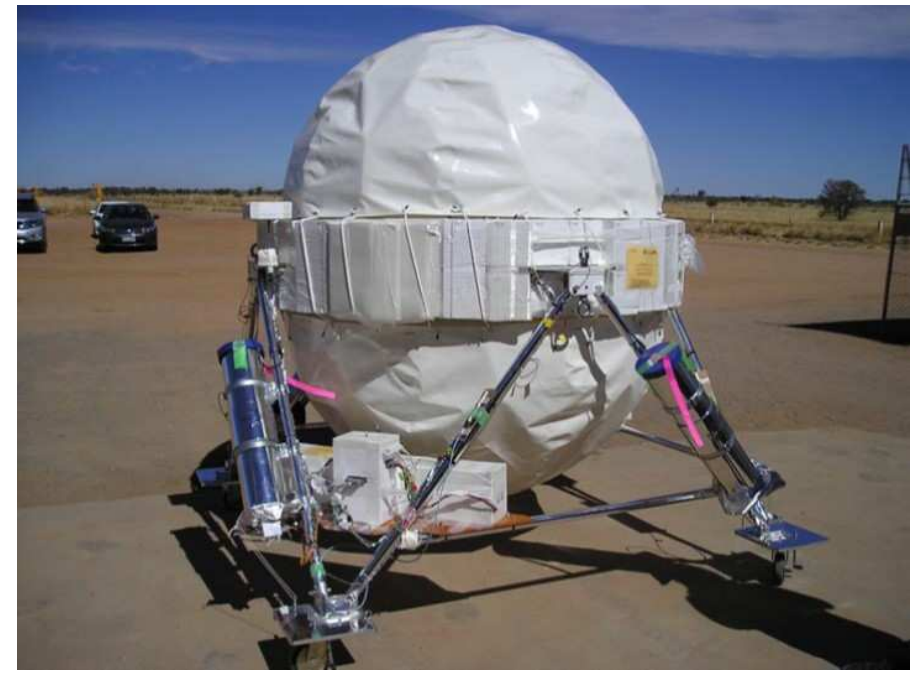

Figure 2: GRAINE-2015 balloon-borne gondola before launching. One of three star cameras was set on the opposite in this picture.
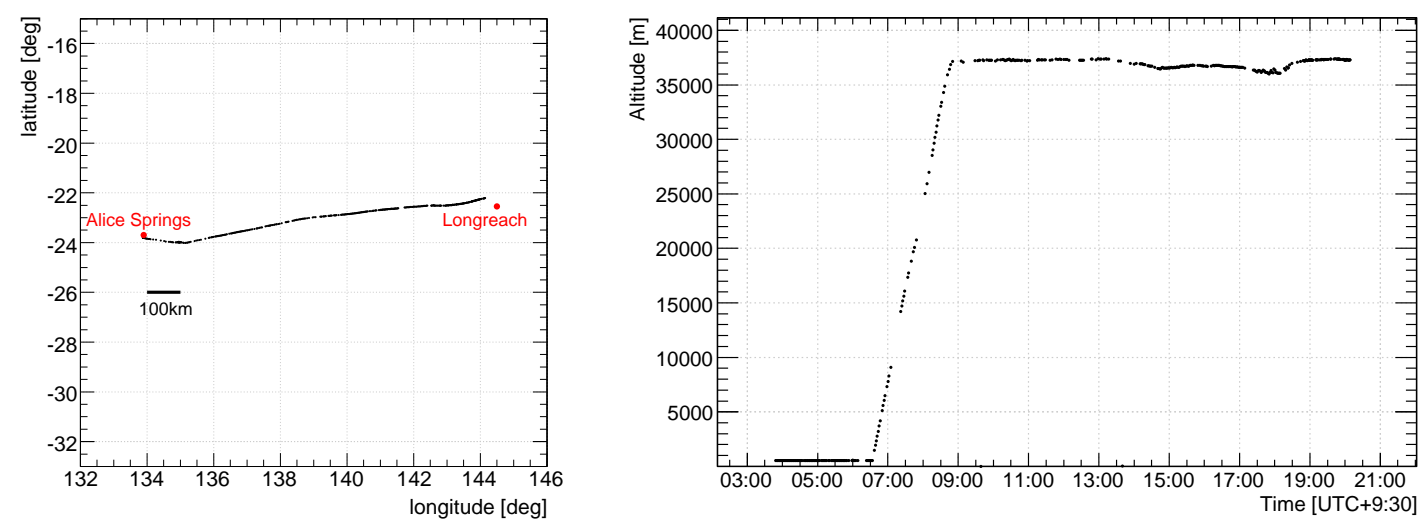

Figure 3: (left) Flight path of GRAINE-2015 balloon. (right) Time variation of balloon altitude.

nearby Longreach at around 20:55. Total flight duration was 14.4hours and level flight duration was 11.5hours. Observation time of the Vela pulsar within telescope's filed of view ( \pm 45 degree in zenith angle) was from 14:00 to 20:02. The payload including emulsion chambers and SSD was recorvered successfully in 13th May at around 14:00.

\section{Flight data analysis of the emulsion film}

\subsection{The emulsion film development}

Recovered emulsion films were transported from Longreach to the university of sydney by land route in keeping cool. We have constructed the development facility in the sydney university. All emulsion films were developed at there between 20th May and 1st June. 


\subsection{Readout of the emulsion tracks}

Developed emulsion films were transported to Japan. Track infromation in emulsion were read out by automatic emulsion scanning system, $\mathrm{HTS}^{1}$ at Nagoya university. HTS is next generation scanning system, enable us to read out at $0.9 \mathrm{~m}^{2} /$ hour (100 times faster than the previous system). We are currently tunig about the readout parameters finely. In this time, scanning area was set in one $12 \mathrm{~cm} \times 8 \mathrm{~cm}$ region of an emulsion film with $\left|\tan \theta_{\text {proj }}\right| \leq 2.0$ angle acceptance.

\subsection{Reconstruction performance of the emulsion tracks}

The track detection efficiency of one emulsion film was evaluated by using penetrated tracks in the emulsion films. Figure 4 shows the one of the evaluated result about the track detection efficiency in one $12 \mathrm{~cm} \times 8 \mathrm{~cm}$ region of an emulsion film (Converter-unit4, \#95). The track detection efficiency is $>95 \%$ with uniformity for all incident angle and all scanning area.
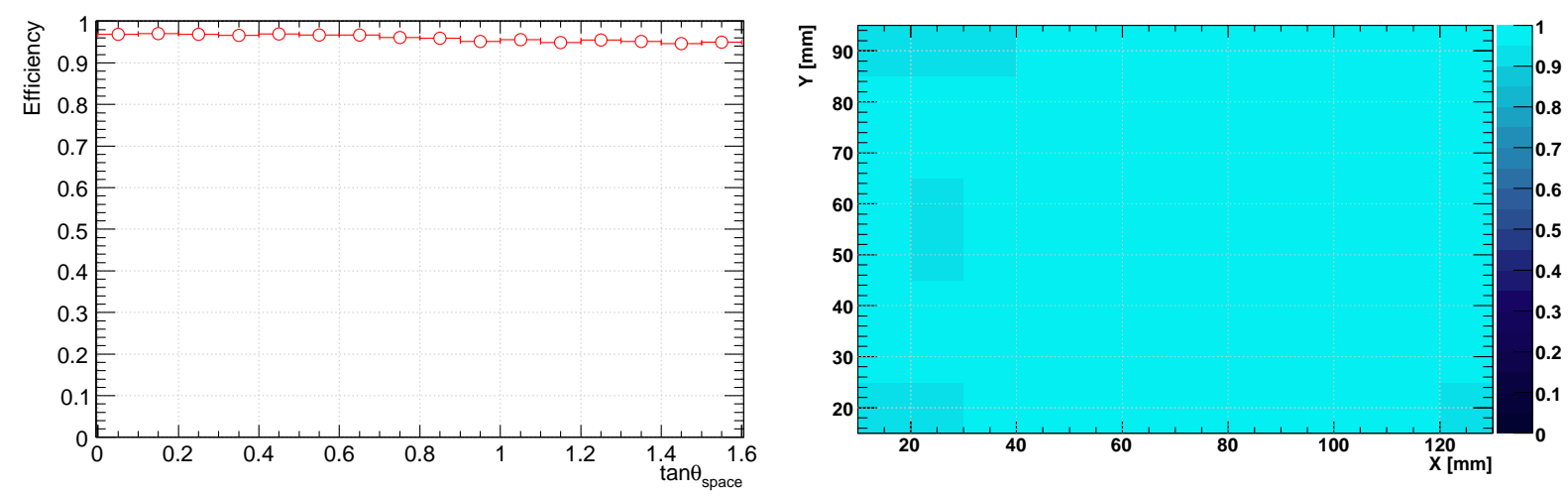

Figure 4: (left) The track detection efficiency as a function of incident angle. (right) The position distribution of the track detection efficiency in one $12 \mathrm{~cm} \times 8 \mathrm{~cm}$ region of an emulsion film.

The track connection accuracy was also evaluated by penetrated tracks. Figure 5 shows the track position and angle difference for X-projection, fitted with a Gaussian function. The values of $\sigma$ obtained by fitting with a Gaussian were $0.89 \mu \mathrm{m}$ and $4.2 \mathrm{mrad}$, respectively.

\section{Flight data analysis of the attitude monitor}

\subsection{Operation status}

There are some troubles about the daytime star camera. One of three cameras (SC1) was stopped due to the hardware error at around 11:30. Another star camera (SC2) was stopped due to the software problem at around 15:10.

Figure 6 shows the images which were taken by the SC3. Figure 7 shows the time variation of averaged brightness value in one frame about the SC3. When the brightness value was saturated, the star camera was affected by the sunlight or the stray light.

\footnotetext{
${ }^{1}$ Hyper Track Selector
} 

Keita Ozaki ${ }^{1}$
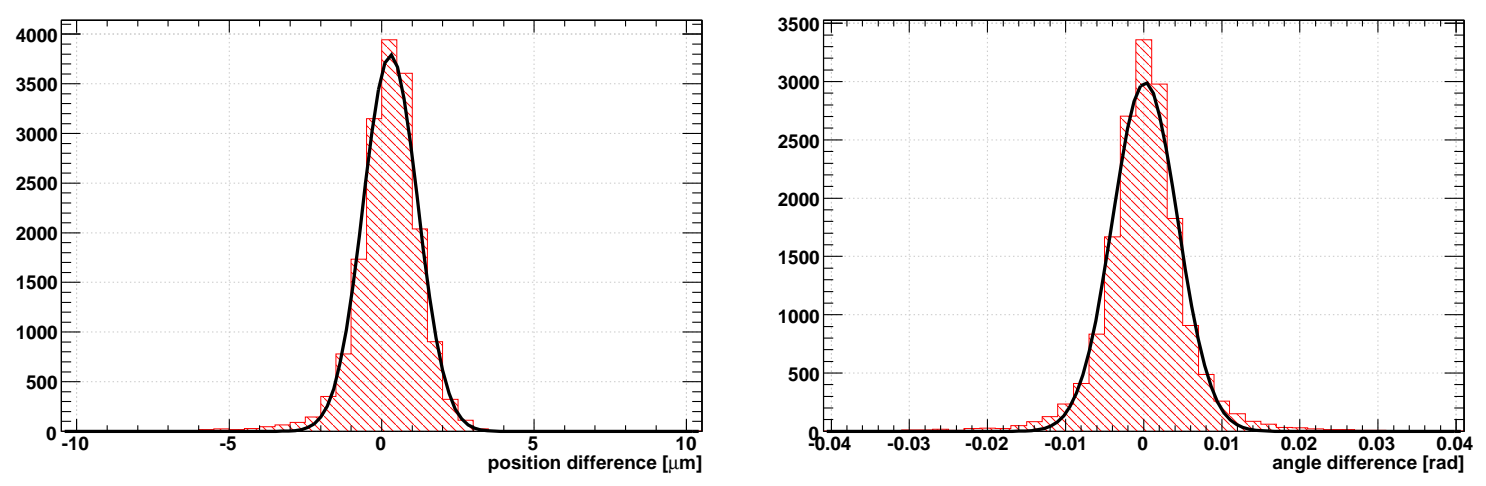

Figure 5: (left) Distribution of position difference for X-projection. (right) Distribution of angle difference for X-projection.
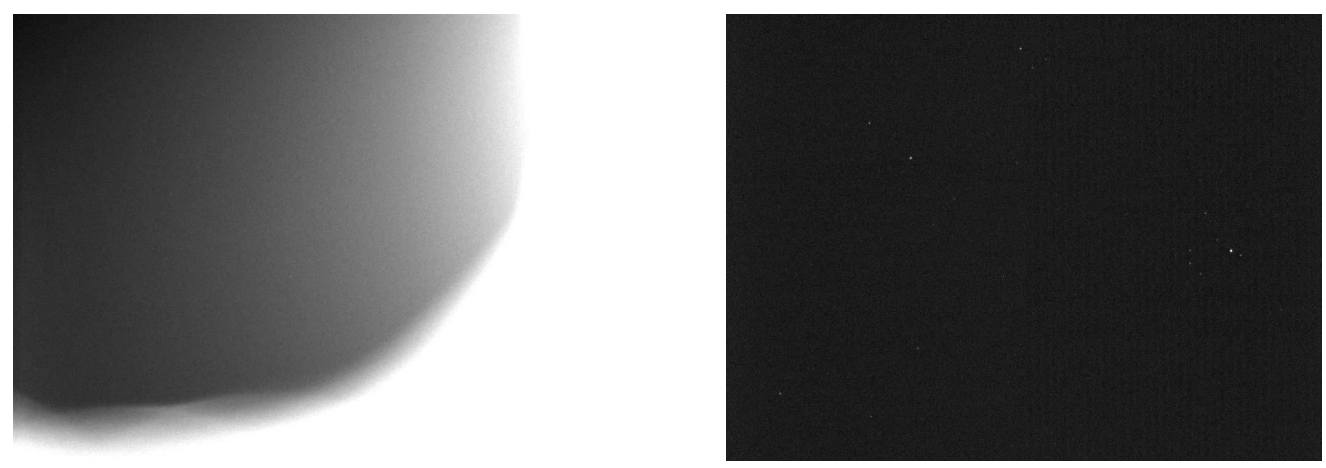

Figure 6: (left) This image was taken at 17:01:54.38 at balloon altitude. (right) This one was taken at 18:46:48.38 at balloon altitude.

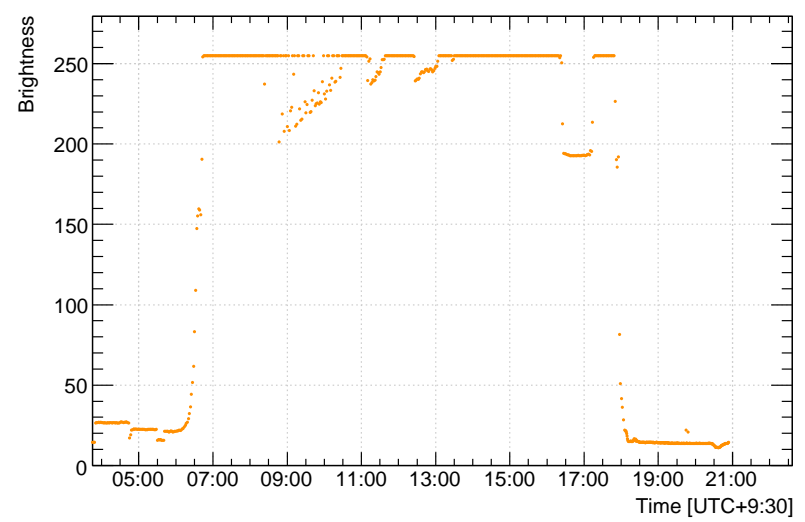

Figure 7: The time variation of averaged brightness value of the star camera No.3. 


\subsection{Attitude determination}

We are analyzing the captured images to determine the attitude information. At first, star-like pixel were selected if the pixel brightness has an excess over the threshold. The captured images, which have the bright components by the stray light, were applied image processing filter to clean up the bright components. A star-image was spreading plural pixels, so their pixels were clustered, and the center position of star-image was calculated by weighted brightness of their pixels. View direction of the star camera was determined by the pattern matching between selected star pattern and starcatalog.

Figure 8 shows the time variation of the azimuth (yaw), elevation (pitch), and rotation (roll) direction determined by the SC3. The accuracy of the attitude determination were sub-arcminute $(\sim 0.001 \mathrm{deg})$ for yaw and pitch direction, and several-arcminute $(\sim 0.05 \mathrm{deg})$ for roll direction. We have determined the attitude information in a period of time from 17:50 to 20:05. We will analyze the rest of the live time.
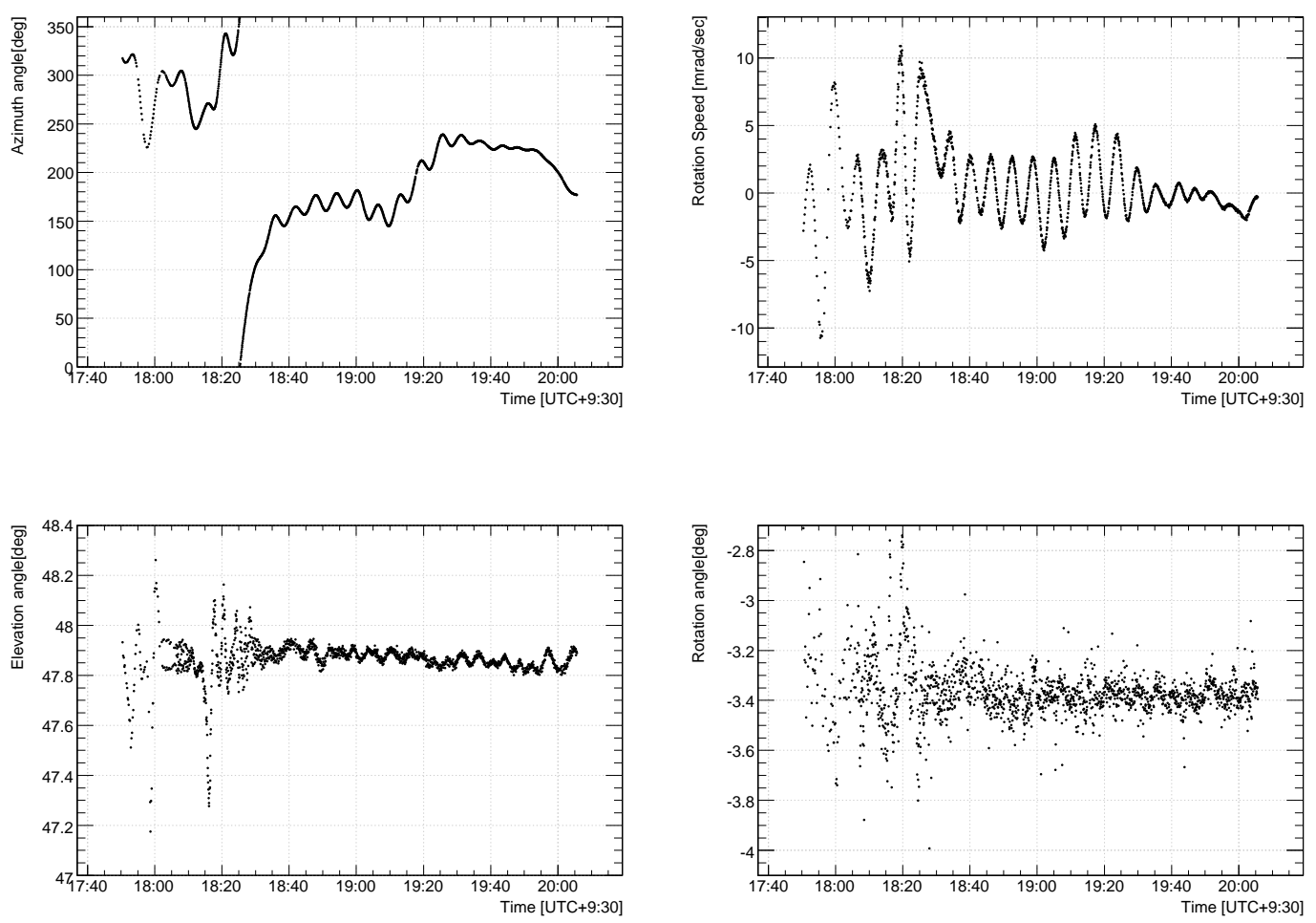

Figure 8: The time variation of the yaw (top left), pitch (bottom left), and roll (bottom right) direction. (top right) Rotation speed of the balloon gondola.

\section{Summary}

GRAINE is a balloon-borne emulsion $\gamma$-ray telescope experiment to observe cosmic $\gamma$-ray with precise angular resolution and polarization sensitive, covering the energy range from $10 \mathrm{MeV}$ 
to $100 \mathrm{GeV}$. The second balloon-borne experiment was performed at Alice Springs in Australia in 12th May 2015. The balloon flight and recorvery of the payload were went well. Now we are analyzing the flight data of the emulsion chamber. The detection efficiency of emulsion tracks and the accuracy of the track connection were evaluated by reconstruction of tracks between emulsion films. We are also analyzing the flight data of the star camera as an attitude monitor. The godola's attitude of the last half of level flight were determined by star camera No.3. The attitude determination accuracy is better than the requirement value from the $\gamma$-ray telescope. The detection of the vela pulsar and the demonstration of the $\gamma$-ray telescope's angular resolution will be done to one year.

\section{Acknowledgement}

We would like to thank the staff of the ISAS/JAXA, CSIRO and the University of NSW at Alice Springs. The scientific balloon(DAIKIKYU) flight opportunity was provided by ISAS, JAXA. We would like to also thank A. Bakich and K. Varvell, the staff of the University of Sydney. This work was supported by JSPS KAKENHI (Grant Numbers 20244031 and 26247039), a Grant-inAid for JSPS Fellows and the Sasakawa Scientific Research Grant from The Japan Science Society.

\section{References}

[1] The Fermi-LAT Collaboration, [astro-ph.HE/1501.02003]

[2] S. Takahashi et al., PTEP, (2015), 043H01

[3] S. Takahashi et al., in the proceedings of 34th ICRC, (2015)

[4] S. Takahashi et al., NIM A 620 (2010) 192.

[5] H. Rokujo et al., NIM A 701 (2013) 127.

[6] H. Rokujo et al., in the proceedings of 34th ICRC, (2015) 